\title{
Risk Assessment Tool based on Demographic Risk Factors to Predict Breast Cancer Risk using Neuro-Fuzzy Technique
}

\author{
Donia S. Al-Tai \\ Department of Computer Science, \\ College of Science, university of \\ Basra, IRAQ
}

\author{
Kareem R. Hassan \\ Department of Computer Science, \\ College of Science, university of \\ Basra, IRAQ
}

\begin{abstract}
In recent years, the statistics presented increased incidence rate of breast cancer, while early detection in early stages considered one of the highest healing rates and also role of prevention in reducing risk by avoiding causes of incidence that associated with risk factors. as well as, The soft computing approaches have been used widely in solving health care problems by modeling to the behavior of experts. For these reasons and others, we presented in this paper the proposed method is designing a risk assessment tool to prevention and early detection of breast cancer based ondemographic risk factors (DRF) by using neuro-fuzzy system technique (NF), in order to solve the main problem for this research and help doctors or patients in the risk assessment of incidence. This method consisted of three stages (statistical study, prepare data and design of the assessment model using NF by two approaches, In the second approach which uses the FCM algorithm with NF technique, was proposed to improve the first approach, which used NF technique. also achieved a higher accuracy for results than the other tools (Gail,IBIS). Where rate of success for the proposed tool is $94 \%$. In addition, used MATLAB 2013 to programming and testing the proposed method.
\end{abstract}

\section{General Terms}

Artificial Intelligence, Breast Cancer

\section{Keywords}

Neuro-Fuzzy Systems, Fuzzy C-means clustering, fuzzy logic, breast cancer, statistical study.

\section{INTRODUCTION}

In Iraq and all the world, people are suffering from limited medical resources as well as, long waiting time for get medical services. According to the World Health Organization (WHO), Iraq is leading countries that suffering lack at health care systems [1]. Breast cancer is most common cancers among women, Its malignant tumor that develops from the uncontrolled growth of cells in the breast and then spread to other parts of the body [2]. the cause of the breast cancer is unknown exactly, but is most likely be due to genetic and environmental factors called demographic risk factors. A risk factor is anything that affects your chances of disease incidence, such as cancer. But exist the risk factors do not mean that disease is confirmed. Furthermore it. Some risk factors for breast cancer cannot be changed. For example, family history, age ....etc. Therefore, these factors considered as a way to reduce risk of incidence by risk assessing [3].

The risk assessment applied for predict to future about disease result, which is considered as indicator of early detection and healing. Statistical methods is most widely used in the analysis of risk assessment data but recently, artificial intelligence techniques considered efficient alternative methods $[4,5]$ and helped solve health care problems through the development of expert systems to reduce cost, waiting time, and errors begining by medical staff. CAD has become one of the leading research areas in medical diagnostic aimed to designing an expert system that combines human experience and artificial intelligence techniques to achieve a more accurate diagnosis [6].

In the medical field, artificial neural networks are most important, especially after the discovery Back-propagation algorithm (BP) ,which became very common in medical prediction. In order to overcome the limitations of artificial neural network (ANN) combines with Fuzzy Inference System (FIS) that provides ability to convert data into a knowledge that people understand using the fuzzy set to represent and process the data and information in which exist them inaccuracy $[7,8]$.

In this paper, the hybrid system is presented, which includes fuzzy logic (FL) and neural networks (NN), called neurofuzzy system (NF), To overcome some of the disadvantages of individual approaches, such as the Black Box for Neural Networks (NN) and Learning capability to fuzzy Systems (FL) [9]. And then used it to predict the risk based on demographic risk factors in risk assessment for each person. In addition, improving Neuro-fuzzy system by using FCM algorithm, which adjusts on fuzzy part parameters of system by adjusting the rules and parameters automatically to overcome the disadvantage of grid partition method, which depends experts in determining rules and dividing inputs space based on their experience. This process often takes a lot of time when designing rules. There are many methods, but FCM is most used when dealing with medical data [10].

\section{BACKGROUND}

\subsection{Prevention and Early detection of Breast cancer}

Global and local statistics of breast cancer illustrate It is most common cancer in the world and forms about $23 \%$ of cancers [11]. In Iraq, is the most common cancer among the Iraqi people and represents third of cancer cases registered in women, according to record of cancer in Iraq 2011. While the studies in Basra presented that about 2,123 new cases of breast cancer in females diagnosed during the period 2005 2012 [12]. The results of these studies illustrate increasing effort to establish programs to Fighting breast cancer in Iraq. The main objective of these guidelines is to reduce deaths. Simple instructions for initial prevention from these malignant tumors, called Fighting cancer programs, included following methods of cancer control proposed by the WHO [13]: 
- Prevention: Attempts to reduce or eliminate exposure to cancer-causing factors by changing lifestyle or various forms of active intervention for example experiment the drug (Tamoxifen) [14].

- Early detection and screening: Increased awareness of signs and symptoms of cancer contributes to early diagnosis. Also, screening for healthy individuals where treatment is effective [15].

The causes of breast cancer are still unknown, and there is no certain way to prevent it, while recent studies most likely to be genetic and environmental factors called demographic risk factors, that can reduce risk and help increase the early detection and the treatment chance. Many women have risk factors but do not develop breast cancer for them, but these factors identify women who benefit most from screening and preventive measures. These factors are internal uncontrollable and include (age, sex (gender), puberty, age of first child, menopause, family and personal history, genetic risk factors, number of children, early breast radiation and breast density), or external factors controllable related to lifestyle include (Obesity, Breastfeeding, Hormone Therapy (HRT), Contraceptive Pill (OCP), Alcohol Consumption, Physical Activity) $[14,15]$.

Early detection of breast mass in healthy women before to be clear is a way to save lives. Therefore, screening should be performed where all women are at risk of breast cancer. This risk varies from (high, median or low) according to risk factors [14]. Cancer control and breast screening programs having a major role in the early detection to most cases detected in developed countries during the 20th century. Thus, breast cancer screening programs have significant effects in early diagnosis of the disease [16].

\subsection{A brief overview about Artificial Intelligence Techniques used}

Recently, become uses of various artificial intelligence techniques (AI) in medicine more popular and the motivation for this work to investigate whether the use of artificial intelligence techniques in predicting of risk can improve the predictive capacity of breast cancer risks as in other cancers. And then comparison predictive capacity between artificial intelligence techniques and other statistical methods. This section provides a brief overview of artificial intelligence techniques (AI) that will be used for proposed method in this paper $[17,18]$.

\subsubsection{Fuzzy logic}

Fuzzy logic theory (FL) proposed by Lotfi Zadeh in 1965 to represent and process data and information in which contains uncertainty. fuzzy logic analyzes information using fuzzy sets (FS) by forming of linguistic expressions rather than numeric values and using IF-THEN to connect them and form a series of linguistic rules that describe behavior of system through knowledge of human expert [19].

Figure 1 shows three basic stages of fuzzy logic system that represent steps of fuzzy inference. The Fuzzification component is process convert crisp input values to the appropriate fuzzy input values in range between $(0 . .1)$ using the specified membership function. Inference Engine component is process human decision-making based on fuzzy concepts to obtain fuzzy outputs by embedding the fuzzy values obtained from fuzzification step in IF part from rule and then using event operator(AND) to aggregate the output values. Defuzzification component transforms the fuzzy output into a crisp output by applying one of defuzzification methods, often use center-of-gravity (COG) method [20,21].

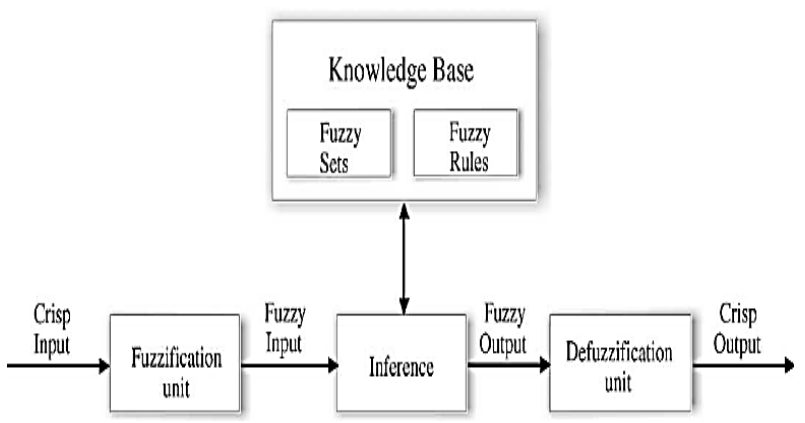

Fig 1: Fuzzy Inference System

\subsubsection{Fuzzy c-means Algorithm}

Fuzzy c-means algorithm is clustering method that allows for one part of data belong to several clusters at the same time with different degrees of membership, instead of belonging to one cluster. This algorithm improved by Bezdek in 1981 and is clustering method with supervisor based on reduction of objective function according to equation (1) [22].

$$
J(U, V)=\sum_{i=1}^{n} \sum_{j=1}^{c}\left(\mu_{i j}\right)^{m}\left(d_{i j}\right)^{2}
$$

The fuzzy partition is used to improve objective function $\mathrm{J}(\mathrm{U}, \mathrm{V})$, with update the degree of membership $\mu \mathrm{ij}$ and clusters centers $\mathrm{vj}$. The algorithm works frequently using equations $(2,3)$ respectively until one of the following stop criteria achieved [23, 24]:

1) difference between updated and previous objective function $\mathrm{J}(\mathrm{U}, \mathrm{V})$ is less than specified minimum threshold.

2) Maximum Iteration is reached.

$$
\begin{gathered}
v_{i}=\frac{\sum_{i=1}^{n}\left(\mu_{i j}\right)^{m} x_{i}}{\sum_{i=1}^{n}\left(\mu_{i j}\right)^{m}} \\
\mu_{i j}=\frac{1}{\sum_{k=1}^{c}\left(\frac{d_{i j}}{d_{j k}}\right)^{\frac{2}{m-1}}}
\end{gathered}
$$

\subsubsection{Neuro-Fuzzy Systems (NFS)}

In artificial intelligence fuzzy logic (FL) and neural networks $(\mathrm{NN})$, each one of them has certain properties that make it suitable for solving a large number of problems. However, they also have some limitations that do not allow for apply them in some cases. To overcome these limitations, artificial neural networks (ANN), which provide the ability to learn integrated with fuzzy systems (FS) that provide ability to perceive to be created hybrid Neuro-fuzzy system (NFS).This approach overcomes limitations, so that neural networks become more clear by adding represent knowledge through integrating it with fuzzy systems that become capable of learning. The structure of the Neuro-fuzzy system is shown in Figure 2 and is similar to structure of neural network with four layers. The parameter update process by using forward and backward passes [9, 25, 26]. 


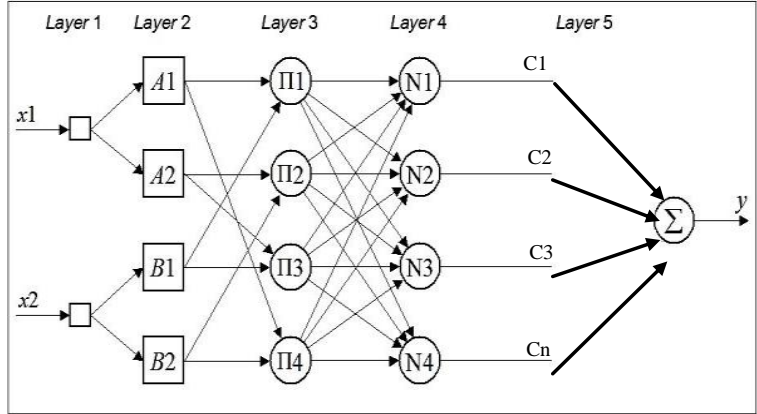

Fig 2: Neuro-Fuzzy System structure

In forward pass, outputs of neuron are calculated layer by layer as follows: Layer $\mathbf{1}$ is Fuzzification layer, each Neuron in this layer performs fuzzifier by determines degree of membership for each input using Gaussian membership function, so that the output values between $(0 . .1)$ and given as:

$$
\mu_{A_{i j}}\left(x_{j}\right)=e^{-\frac{1}{2}\left(\frac{x_{j}-a_{i j}}{b_{i j}}\right)^{2}}
$$

Layer 2 is Rule layer, each neuron in this layer correspond to rule, where receiving input from Layer 1 and calculates the firing strength for IF part of rule by product membership degree of inputs according to rule in this neuron, so that output of this layer is firing strength. Layer 3 is Normalization layer, each neuron receives inputs from Layer 2, and calculates the ratio of the firing strength of a given rule to the sum of firing strengths of all rules. Layer 4 is Defuzzification layer, single neuron determines final output through product each value from layer 3 with corresponding weight to produce the center of gravity in process of defuzzification and output is crisp value $[9,26]$.

In backward pass is a learning and training step for the Neuro-fuzzy system (NFS) in which propagates error signals to update parameters for IF part and THEN part of rule [26]. NFS training can use Back-propagation algorithms to reduce the error of the training (MSE) and then adjust network parameters using training patterns. These parameters can be divided into two categories based on IF part parameters and THEN part parameters of rule. In the IF part the center (a) and width (b) of Gaussian functions are adjusted, whereas THEN part parameters adjusted is weight [27].

In the medical, Neuro-fuzzy approaches have been used in many areas, including in the prediction of disease.

\section{DATA AND METHOD}

\subsection{Data}

A set of breast cancer data collected by Oncology center at Al Sadir Teaching Hospital at Basra were used to model the risk assessment tools to predict risks of breast cancer using NeuroFuzzy system technique.

In this study, the samples of research community that consists 200 patients and 10 doctors at Oncology Center determined. Also using statistical methods, especially descriptive statistics to describe research problem and determine most important demographic risk factors that increase chance of develop breast cancer. The statistical analysis to questionnaires used to collect data through its applying on the sample and use statistical methods to extract most important factors in Basra city and divide the range of each risk factor to High, median or low risk levels, where each level represents the degree of its effect in increase breast cancer risk. This degree was determined by applying the statistical study on oncology center records to determine the levels with large number of incidence. Thus, determine the affect each level in increased risk of develops breast cancer.

Statistical analysis of questionnaires and oncology center records depend on percentages, frequencies, and weighted mean to assessing most effective and influential factors in Basra environment. The result was determined 6 factors is most important. all this factors is internal demographic risk factors that includes age, address, estrogen, number of children, Age at the birth of the first child, family history). Data collected for 200 sample of research community to use in modeling of proposed method. This result obtained from statistical study for period 7 months. Table 1 includes the factors and the range for each factor and the factor partition into categories that represent risk level and its effect on increased risk, also center and width of membership functions.

Table 1. Demographic risk factors and categories

\begin{tabular}{|c|c|c|c|c|c|}
\hline $\begin{array}{c}\text { Input and } \\
\text { output } \\
\text { variables }\end{array}$ & $\begin{array}{l}\text { Linguistic } \\
\text { value }\end{array}$ & \begin{tabular}{|c|} 
Fuz \\
zy \\
sets \\
code
\end{tabular} & Center & Width & Rang \\
\hline \multirow{3}{*}{ Age } & young & $\mathrm{L}$ & 20 & 12.7 & \multirow{3}{*}{$80-20$} \\
\hline & mid of age & $\mathrm{M}$ & 50 & 12.7 & \\
\hline & old & $\mathrm{H}$ & 80 & 12.7 & \\
\hline \multirow{3}{*}{ Address } & $\begin{array}{l}\text { Low-risk } \\
\text { region }\end{array}$ & $\mathrm{L}$ & 0 & 7.6 & \multirow[t]{3}{*}{$36-0$} \\
\hline & $\begin{array}{l}\text { mid -risk } \\
\text { region }\end{array}$ & M & 18 & 7.6 & \\
\hline & $\begin{array}{l}\text { High-risk } \\
\text { region }\end{array}$ & $\mathrm{H}$ & 36 & 7.6 & \\
\hline \multirow{3}{*}{ Estrogen } & $\begin{array}{c}\text { small } \\
\text { duration }\end{array}$ & $\mathrm{L}$ & 11 & 8.4 & \multirow[t]{3}{*}{$51-11$} \\
\hline & $\begin{array}{c}\text { normal } \\
\text { duration }\end{array}$ & M & 31 & 8.4 & \\
\hline & $\begin{array}{c}\text { large } \\
\text { duration }\end{array}$ & $\mathrm{H}$ & 51 & 8.4 & \\
\hline \multirow{3}{*}{$\begin{array}{l}\text { Number of } \\
\text { child }\end{array}$} & few number & $\mathrm{L}$ & 0 & 2.5 & \multirow{3}{*}{$12-0$} \\
\hline & $\begin{array}{c}\text { Average } \\
\text { number }\end{array}$ & $\mathrm{M}$ & 6 & 2.5 & \\
\hline & $\begin{array}{c}\text { large } \\
\text { number }\end{array}$ & $\mathrm{H}$ & 12 & 2.5 & \\
\hline \multirow{3}{*}{$\begin{array}{l}\text { Age at first } \\
\text { pregnancy }\end{array}$} & Small age & $\mathrm{L}$ & 12 & 7 & \multirow{3}{*}{$45-12$} \\
\hline & middle age & $\mathrm{M}$ & 28.5 & 7 & \\
\hline & old age & $\mathrm{H}$ & 45 & 7 & \\
\hline \multirow{3}{*}{$\begin{array}{l}\text { Family } \\
\text { history }\end{array}$} & $\begin{array}{l}\text { Third } \\
\text { degree }\end{array}$ & $\mathrm{L}$ & 1 & 0.4 & \multirow[t]{3}{*}{$3-1$} \\
\hline & $\begin{array}{l}\text { Second } \\
\text { degree }\end{array}$ & M & 2 & 0.4 & \\
\hline & first degree & $\mathrm{H}$ & 3 & 0.4 & \\
\hline \multirow{5}{*}{ Rate of risk } & $\begin{array}{l}\text { Very low } \\
\text { risk rate }\end{array}$ & $\begin{array}{l}\mathrm{V} \\
\mathrm{L}\end{array}$ & 0 & 0.1 & \multirow{5}{*}{$1-0$} \\
\hline & $\begin{array}{r}\text { Low risk } \\
\text { rate }\end{array}$ & $\mathrm{L}$ & 0.25 & 0.1 & \\
\hline & $\begin{array}{l}\text { Medium } \\
\text { risk rate }\end{array}$ & M & 0.5 & 0.1 & \\
\hline & $\begin{array}{r}\text { High risk } \\
\text { rate }\end{array}$ & $\mathrm{H}$ & 0.75 & 0.1 & \\
\hline & $\begin{array}{l}\text { Very high } \\
\text { risk rate }\end{array}$ & $\begin{array}{l}\text { V } \\
\mathrm{H}\end{array}$ & 1 & 0.1 & \\
\hline
\end{tabular}




\subsection{Pre-processing}

Pre-processing is a process that converts the raw inputs and outputs (target values) into a form understandable or acceptable before the training process. Often, this is used to reduce the dimensionality of input data and to optimize the generalization performance [28].

In order to be used within the NFS model, All data obtained from statistical study must pre-processed. Data were collected for 200 cases and assessment each case by expert to determine desired outputs and then used to modeling proposed method. the expert assessment is linguistic values and NFS model used numerical values in modeling due to this drawback we suggested build fuzzy expert system that used to convert linguistic values into numerical values.

The expert system based on the knowledge obtained from statistical study along with expert's experience in build fuzzy rules and that equal 729 rule to describe the behavior of system, the relationship of variables to each other and their effect on probability of incidence. Also use Gaussian functions to represent risk levels and other parameters as shown in Table I. finally, Outputs are converted to numeric values to be used in assessment proposed model.

In order to Pre-processing to data using expert system, at each time data of each case is entries to system, which represents age, address, duration of stay with estrogen, number of children, the age of the first child and the family history to obtain output value that represent risk rate by performing fuzzy inference process that include (Fuzzification, Evaluation rules, Defuzzification) as shown in Figure 1 and then comparing the output of the system with the evaluation of doctor to ensure the validity of system.

\subsection{Proposed Risk Assessment approach}

Observed through Statistical study that breast cancer is the most common disease for women in Basra, in order to reduce incidence this disease that referred to their causes genetic and environment factors called demographic risk factors. we suggested to build a tool based on these factors to assess the risk of breast cancer. The main goal for this method is prevention by reducing incidence and assist to early detection of breast cancer.

In this study, there are six demographic risk factors obtained from statistic0al study include age, address, estrogen, number of children, first child's birth, family history. The expert uses these factors to risk assessment, so proposed approach takes the same factors as input variables with one output for risk assessment. As shown in Figure 3 that illustrates the general structure of the proposed method.

In this paper presents two approaches proposed for assessment model. Both approaches use the NFs structure to build assessment model. In the first proposed approach propose used NFs structure that represent collected knowledge through statistical study in fuzzy rule and also adjust parameters of fuzzy system by using neural network in the training process. The training process uses back-propagation algorithm are learning method with supervisor and requires that data used (Training and testing patterns) in network consists of inputs (X) desirable output (Yd). input (X) obtained through statistical study by data collection form, while the desired output (Yd) obtained through pre-processing the data by expert system.

The second approach proposes to improve first approach via using FCM algorithm with NFs structure to build assessment model. Fuzzy sets and initial membership functions (MF_FCM) and their centers are identified using FCM algorithm to before training process at network.

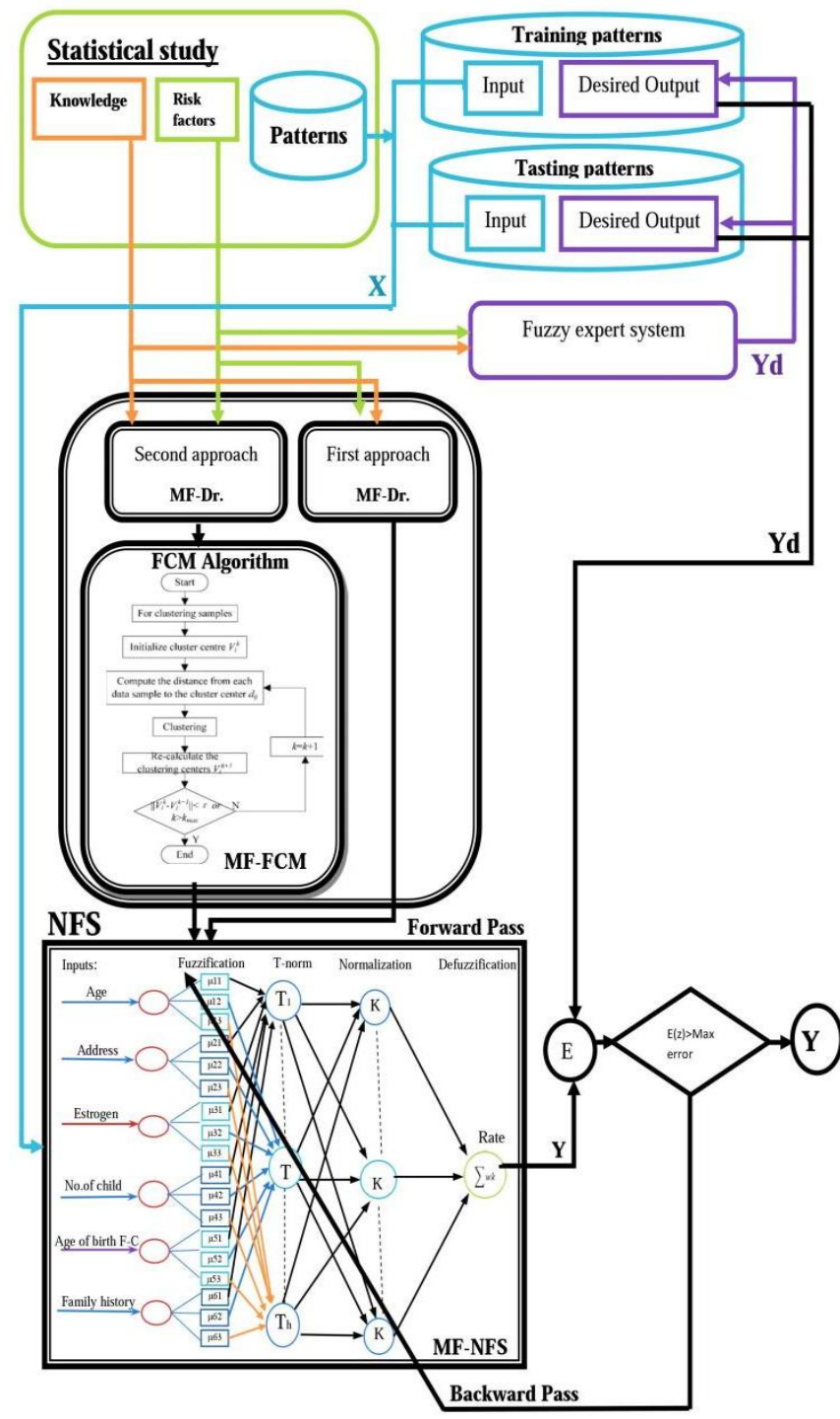

Fig 3: The block diagram of the proposed method

\section{EXPERIMENTAL RESULTS}

The proposed method, data of 200 breast cancer patients were subjected to the pre-processing described above before being passed to the training process for represent training patterns with 150 cases and test patterns with 50 cases. This section presents two proposed approaches to design of assessment model and compares between them to determine the best approach.

In designing risk assessment model the Neuro-fuzzy system is used as first proposed approach and then improving this approach by modeling input and output membership functions using FCM algorithm as second proposed approach. Fuzzy cmeans algorithm uses training patterns to determine the optimal initial centers values of input and output membership functions.

Both approaches use three Gaussian membership functions for each input and back-propagation algorithm to learning. The training options were selected experimentally as follows, 1000 
epochs used to stop the training process and step size is 0.1 . Improve of NFS will be showed by two experiments that representing the results of first approach without using FCM and the results of second approach after improving NFs using FCM algorithm

For first approach, initial parameters of fuzzy inference system must be determined by expert before begin the training process and then membership functions to each input are experimentally adjusted by trial and error. The performance measure (mean square error (MSE)) used to ensure the model did not exceed maximum error limited in network as shown in Figure 4 that shows a decrease in error of network with increase epochs. As well as adjust the parameters of membership function, which represent center and width that shown in Figure 5 and Figure 6 to illustrates membership functions before and after training process, observed 1000 epoch.

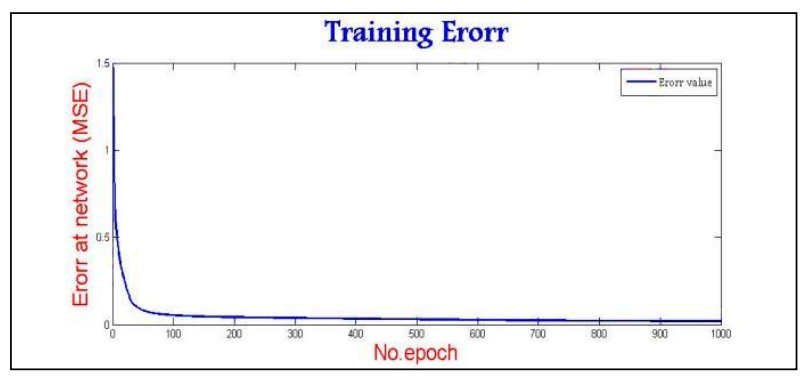

Fig 4: Error versus number of epochs to first approach
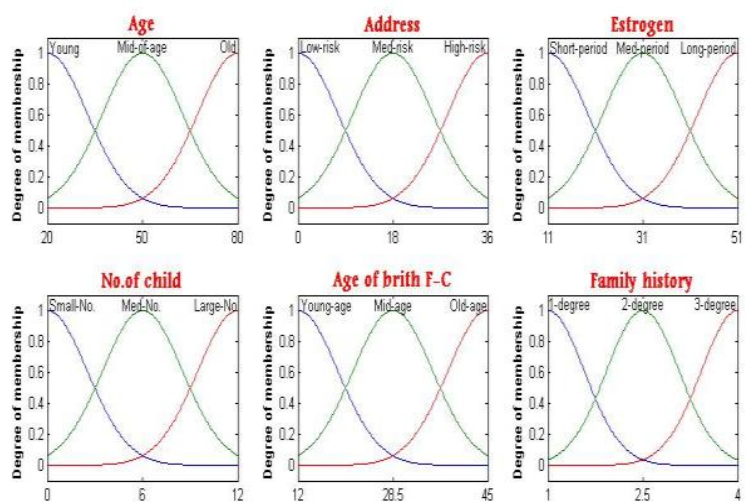

Fig 5: Membership functions before training of first approach
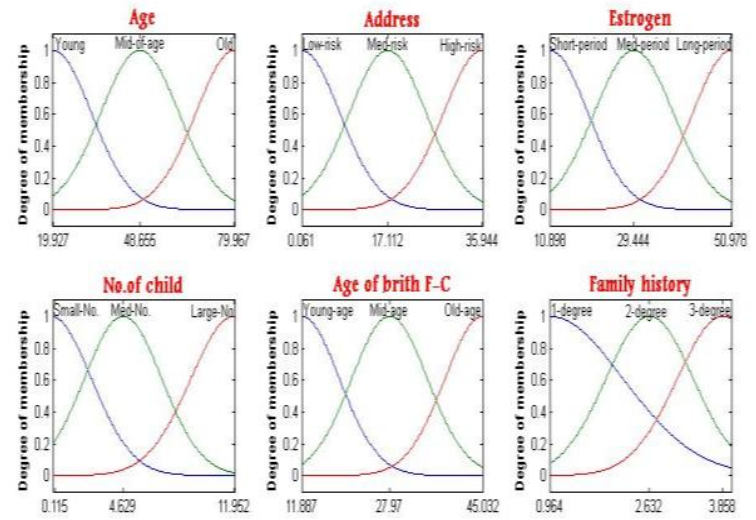

Fig 6: Membership functions before training of first approach
As for the second approach, this uses same NFS parameters in first approach but with using FCM algorithm, which automatically adjusts the parameters before the training process. Figure 7 illustrates membership functions before and after using FCM algorithm, while Figure 8 shows membership functions of second approach after training process and after using FCM algorithm. Also Figure 9 shows the error of network at second approach.
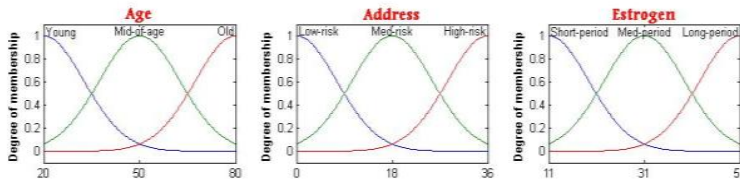

Before
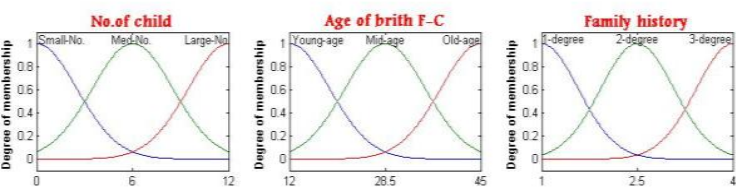

(FCM
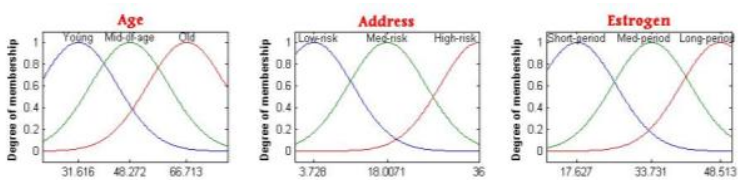

After

(FCM)
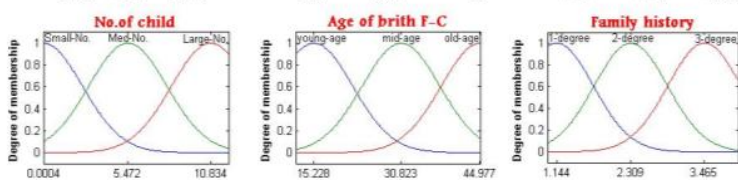

algorithm

Fig 7: Membership functions before and after FCM to second approach
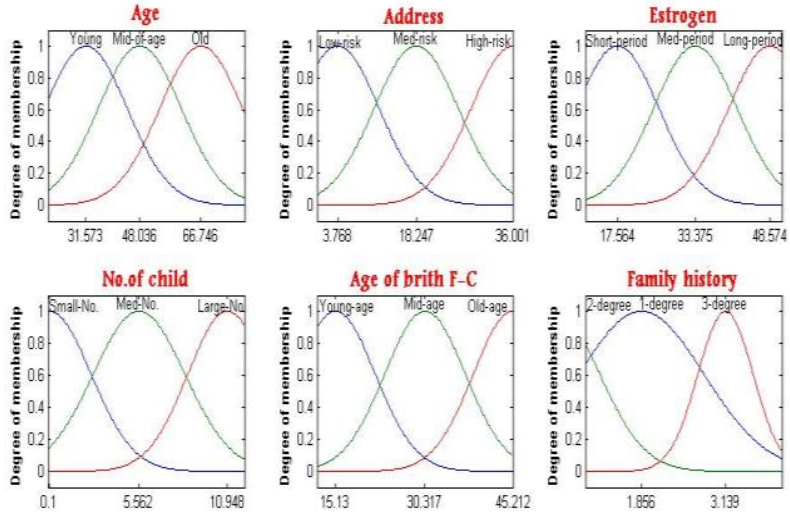

Fig 8: Membership functions after training of second approach

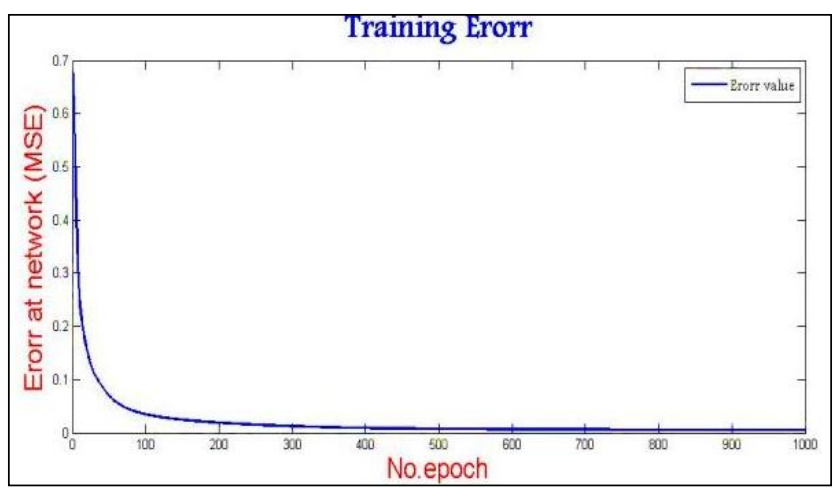

Fig 9: Error versus number of epochs of second approach 
Finally, three final membership functions were selected for each input. Observed that these functions provide a smooth function with results closer to the desired results and minimum error with second approach. Thus, they assigned for the assessment model.

After training process, the network is tested through use testing patterns with final parameters that accessed at training process. Figure 10 shows testing results of first approach by matching desired outputs with the actual outputs for network. Figure 11 shows testing results of second approach. Observe that second approach has a better performance in terms of training and testing as illustrates in Table 2, which shown performance comparison between two approaches.

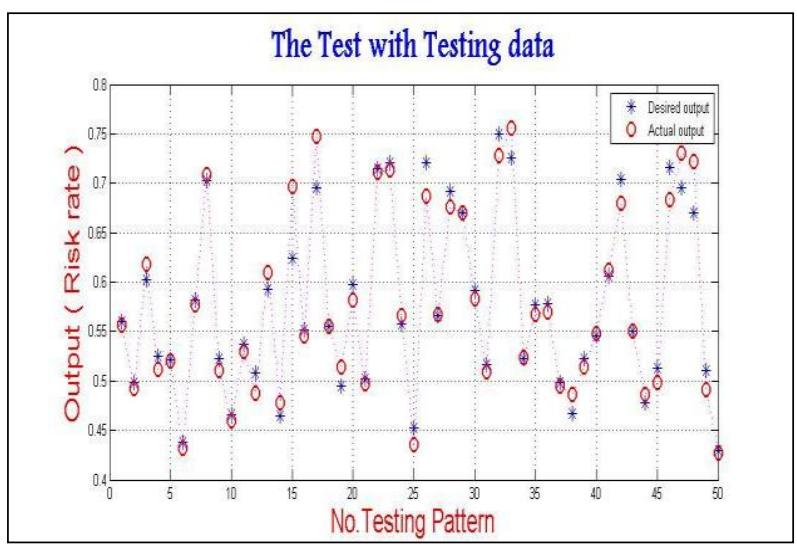

Fig 10: actual output and desired output of first approach

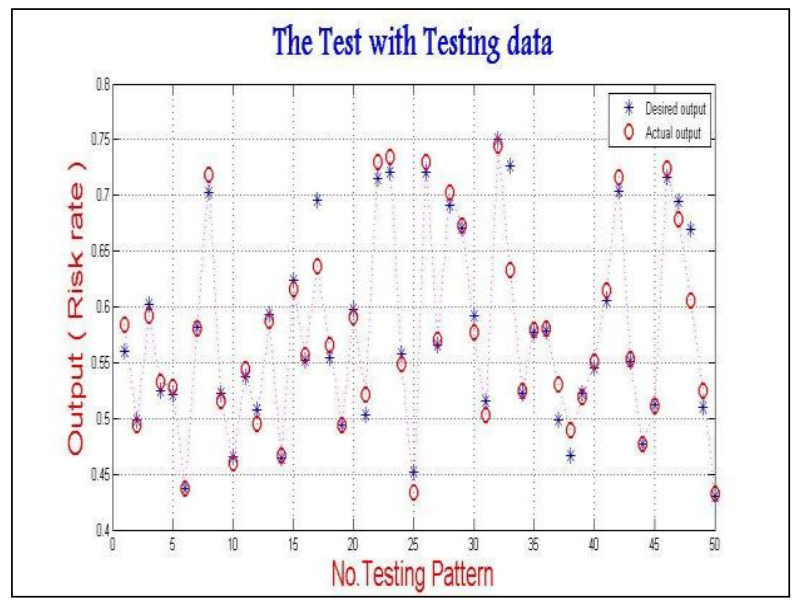

Fig 11: actual output and desired output of second approach

Table 2. Compare the performance of two approaches

\begin{tabular}{|c|c|c|c|c|c|c|c|}
\hline Center $(\eta \sigma)$ & & idth (l|b) & Wight & (१c) & Centers & & o.epoch \\
\hline 0.9 & & 0.2 & 0.0 & & 3 & & 1000 \\
\hline \multirow{2}{*}{\multicolumn{2}{|c|}{$\begin{array}{c}\text { Proposed } \\
\text { approaches }\end{array}$}} & \multicolumn{3}{|c|}{ Training } & \multicolumn{3}{|c|}{ Testing } \\
\hline & & Data & Error & Time & Data & Error & Time \\
\hline \multicolumn{2}{|c|}{$\begin{array}{c}\text { First approach } \\
\text { (NFS) }\end{array}$} & 150 Case & 0.0192 & $23.5 \mathrm{sec}$ & 50 Case & 0.0026 & $0.47 \mathrm{sec}$ \\
\hline \multicolumn{2}{|c|}{$\begin{array}{l}\text { Second approach } \\
\text { NFS with FCM }\end{array}$} & 150 Case & 0.0049 & $22.3 \mathrm{sec}$ & 50 Case & 0.0049 & $0.33 \mathrm{sec}$ \\
\hline
\end{tabular}

The proposed method obtained a high accuracy results compared to other tools. Table 3 shows accuracy of the proposed tool and its comparison with other tools used in risk assessment.

Table 3. Comparison a suggested tool with other tools

\begin{tabular}{|c|c|c|c|}
\hline Models & Difference with Dr. & Correspond with Dr. & Accuracy \\
\hline Gail & 38 Case & 12 Case & $\% 24$ \\
\hline IBIS & 34 Case & 16 Case & $\% 32$ \\
\hline NFS_FCM & 3 Case & 47 Case & $\% 94$ \\
\hline
\end{tabular}

\section{CONCLUSION}

In this paper, main problem is discussed that represent increasing rates of breast cancer in Basra. In order to reduce incidence of $\mathrm{BC}$ through prevention and early detection. We proposed designing a risk assessment tool to predict breast cancer risk based on demographic risk factors (DRF) and using Neuro-fuzzy system technique. The proposed method included three phases for design risk assessment tool is the statistical study, data pre-processing and design of assessment model that include two approaches to processing.

In the first approach, (NF) technique was used to build assessment model. Either the second approach is proposed to improve the first approach by using an algorithm (FCM) with (NF) to adjust the parameters. Thus second approach achieved better results in terms of time and accuracy in the assessment observed from training and testing results. Finally, this approach was adopted in design of assessment tool, and by comparing performance of proposed assessment tool with other assessment tools, we obtained high accuracy to results.

Given the representation of inference systems, it is hoped that such a technique may be better in treat and considered a way to assist professionals without expert.

\section{REFERENCES}

[1] Anderson, Gerard F., et al. (2006). "Health care spending and use of information technology in OECD countries". Health Affairs 25(3), 819-831.

[2] National Cancer Institute. (2007). "Cancer terms", U.S. Department of Health and Human Services, National Institutes of Health. URL: https://train ing.seer.cancer.gov/disease/c ancer/terms.html/ Accessed (2016).

[3] American Cancer Society. "What Is Cancer", Atlanta, Ga: American Cancer Society. URL: https://www.cancer.org/ cancer/cancer-basics .html Accessed (2016).

[4] Lisboa, Paulo JG, Alfredo Vellido, and José David Martín-Guerrero. (2010). "Computational Intelligence in biomedicine: Some contributions". ESANN, 429-438.

[5] Vellido, Alfredo, Elia Biganzoli, and Paulo JG Lisboa. (2008). "Machine learning in cancer research: implications for personalised medicine". ESANN, 5564.

[6] Giarratano, J. and G. Riley (2004), "Expert Systems Principles and Programming". 4ed. Vol. 1. , Boston: PWS Publishing Company. 
[7] Cruz, Joseph A., and David S. Wishart. (2006). "Applications of machine learning in cancer prediction and prognosis". Cancer informatics 2,59.

[8] Lisboa, Paulo JG. (2002). "A review of evidence of health benefit from artificial neural networks in medical intervention", Neural networks 15(1),11-39.

[9] EMAD S. JABBER AL-SHAWI. (2003). "On Multiple Neuro-Fuzzy Systems For Function Approximation". M.Sc. Thesis, department of computer Science, Collage of Science, Basra University.

[10] Cintra, Marcos Evandro, H. A. Camargo, and Maria Carolina Monard. (2008). "Astudy on techniques for the automatic generation of membership functions for pattern recognition.". Congresso da Academia Trinacional de Ciências (C3N), Vol. 1, 1-10

[11] World Health Organization. (2012). "International Agency For Research on Cancer GLOBOCAN 2012: estimated cancer incidence, mortality and prevalence worldwide in 2012". URL: http://globocan.iarc.fr/ Default.aspx Assessed (2017).

[12] Al-Hawaz, M. H., et al., (2016). "Epidemiology of Breast Cancer among Females in Basrah". Asian Pacific journal of cancer prevention: APJCP 17.S3, 191-195.

[13] World Health Organization, "Breast cancer burden". URL: http://www. who.int/cancer/detection/breastancer/ en/index1.html/ Assessed (2016).

[14] National Cancer Institute, NIH Senior Health: " Breast Cancer FrequentlyAskedQcan". Available at: https://nihseniorhealth.gov/breastcancer/breastcancerdefi ned/01.html/ Accessed (2016).

[15] American Cancer Society. "Breast Cancer". URL: http://www.cancer.org/acs/groups/cid/documents/webcon tent/003090-pdf.pdf. Assessed (2016).

[16] Alwan, N. A. S., et al., (2012). "Knowledge, attitude and practice regarding breast cancer and breast selfexamination among a sample of the educated population in Iraq". East Mediterr Health J; 18(4), 337-45.
[17] Keleş, Ali, Aytürk Keleş, and UğUr Yavuz. (2011). "Expert system based on neuro-fuzzy rules for diagnosis breast cancer". Expert systems with applications 38(5), 5719-5726.

[18] Fatima, Bekaddour, and Chikh Mohammed Amine. (2012). "A neuro-fuzzy inference model for breast cancer recognition". International Journal of Computer Science \& Information Technology 4(5), 163.

[19] Cai, Kai-Yuan, and Lei Zhang. (2008). "Fuzzy reasoning as a control problem". IEEE Transactions on fuzzy systems 16(3), 600-614.

[20] Negnevitsky, Michael. (2005). "Artificial intelligence: a guide to intelligent systems". Pearson Education Limited, Essex, England.

[21] Zadeh, Lotfi A., ed. (2013). "Computing with words in Information/Intelligent systems 1: Foundations". Vol. 33. Physica.

[22] Bezdek, James C. (2013). "Pattern recognition with fuzzy objective function algorithms". Springer Science \& Business Media, New York.

[23] CHEN, M.Y. \& LINKENS, D.A. (2004). "Rule-base self-generation and simplification for data-driven fuzzy models". Fuzzy Sets and Systems, 142, 243-265.

[24] Imad S. Alshawi and Lianshan Yan, China. (2012). "Enhancing Accuracy in NeuroFuzzy Systems Using Fuzzy C-Means Clustering Algorithm". IEEE.

[25] JANTZEN, J. (1998). "Neurofuzzy modeling". Tech rep., Tecnical University of Denmark, Department of Automation.

[26] Wang, Zhenyu, Vasile Palade, and Yong Xu. (2006). "Neuro-fuzzy ensemble approach for microarray cancer gene expression data analysis". Evolving Fuzzy Systems, 2006 International Symposium on, IEEE, 241-246.

[27] S. Seung , (2000) ."Backpropagation Learning". IEEE Trans. Systems, Man and Cybernetics, .

[28] Bishop, Christopher M. (2007). " Neural networks for pattern recognition". Oxford university press, UK. 